\title{
Aluminum Nanoparticles Acting as a Pulmonary Vaccine Adjuvant-Delivery System (VADS) Able to Safely Elicit Robust Systemic and Mucosal Immunity
}

\author{
Ning Wang ${ }^{1} \cdot$ Chunliu Wei $^{2} \cdot$ Zina Zhang $^{2} \cdot$ Ting Liu $^{2} \cdot$ Ting Wang $^{2}$ (1)
}

Received: 7 April 2020 / Accepted: 29 April 2020 / Published online: 9 May 2020

(c) Springer Science+Business Media, LLC, part of Springer Nature 2020

\begin{abstract}
Vulnerability of respiratory mucosa to invasions of airborne pathogens, such as SARS-CoV, MERS-CoV and avian viruses which sometimes cause a life-threatening epidemic and even pandemic, underscores significance of developing a pulmonary vaccine adjuvant-delivery system (VADS). Herein, 30-nm aluminum nanoparticles (ANs), unlike the mostly used adjuvant alum which is unsuitable for delivering pulmonary vaccines due to side effects, proved able to act as a VADS fitting inhalation immunization to elicit wide-spread anti-antigen immunity. In vitro ANs facilitated cellular uptake of their cargos and, after pulmonary vaccination, induced mouse production of high levels of anti-antigen IgG in serum and IgA in saliva, nasal, bronchoalveolar and also vaginal fluids. Besides, IFN- $\gamma$ and anti-antigen IgG2a enriched in immunized mice which meanwhile showed no obvious lung inflammation indicated balanced Th1/Th2 responses were safely induced. These outcomes suggest ANs may be an efficient pulmonary VADS for defending against pathogens, especially, the ones invading hosts via respiratory system.
\end{abstract}

\section{Graphic Abstract}

Aluminum nanoparticles can safely induce humoral and cellular immunity at systemic and mucosal level through pulmonary vaccination to contrast the conventional adjuvant alum.

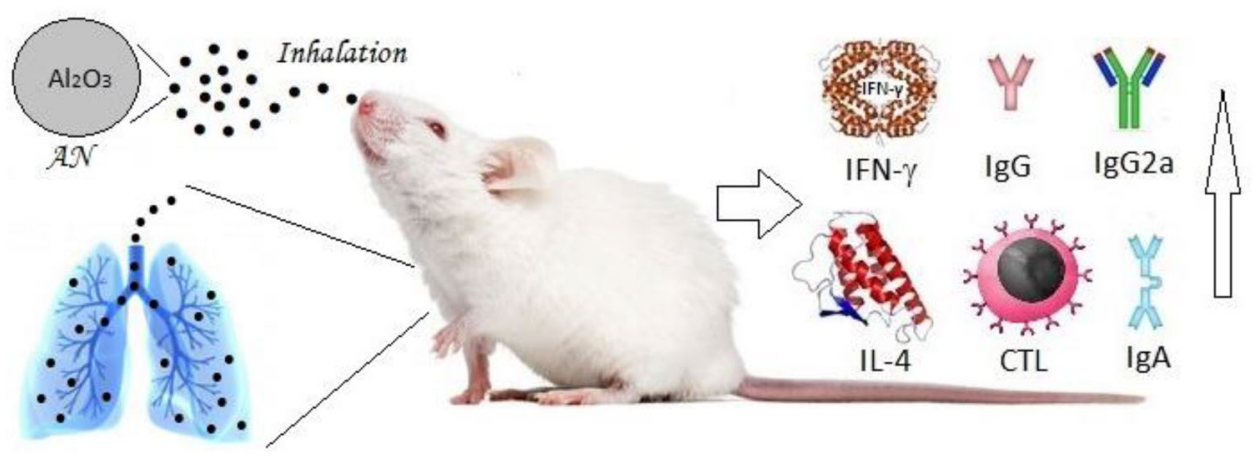

Keywords Nanocarrier $\cdot$ Vaccine delivery $\cdot$ Bioinorganic chemistry $\cdot$ Mucosal vaccination $\cdot$ Cellular immunity

\section{Introduction}

Ning Wang and Chunliu Wei have contributed equally to this work.

Ting Wang

twangcn@ahmu.edu.cn

Extended author information available on the last page of the article
Vaccines play a key role in prevention and control of infectious disease transmission and have saved numerous people's lives since they were developed into industrial products [1, 2]. Therefore, vaccines are argued to be the cornerstone of 
modern public health intervention and regarded as the greatest medical achievement gained by humans [3].

Traditionally, vaccination is performed via subcutaneous or intramuscular injection, both of which prove effective for inducing protective systemic immune responses [4]. However, injection vaccination often shows difficulty in sponsoring the antigen-specific immune responses at mucosa, which is the gate whereby most infectious agents invade hosts [5]. Thus, injection of vaccines can hardly elicit mucosal immunity to form the first line of barrier to pathogen attack. Also, injection involves inconvenience of management, poor compliance, and difficult administration without trained medical professionals [6]. By contrast, mucosal vaccination can be conveniently carried out and demonstrates the ability of triggering systemic as well as mucosal immunity toward pathogens. Moreover, mucosal administration is considered the beneficial vaccination route, because mucosal immune system is evolved with numerous mucosa-associated lymphoid tissues (MALTs) [7]. MALTs contribute to constituting the mucosal immune network throughout the body and function to facilitate setting up comprehensive immunity against invading agents [8]. Mucosal vaccination can be undertaken at mucosa of several physiological and anatomical sites, such as gastrointestinal lumen, nasal cavity, pulmonary alveolus, vagina and oral cavity [9-15].

Respiratory system mucosa represents the most vulnerable and popular site whereby lethal pathogens attack and knock out hosts, because it shows a mild environment and exchanges air and borne materials most frequently with the outside [16]. Recently, several ravaging epidemics or pandemics with a high fatality are all associated with respiratory system infections, e.g., 2003 SARS, 2009 H1N1 swine flu, 2012 MERS, and the now ongoing COVID19 caused by SARS-CoV-2 (severe acute respiratory syndrome-associated coronavirus-2) [17-19]. In particular, SARS-CoV-2 is now still rapidly spreading across the whole world from initial epicenter of Wuhan in China, and has caused a severe pandemic claimed nearly 15000 people's lives among more than 300000 infections [20]. To prevent the epidemic situation worsening, China has taken tough measures on containment of the transmission, such as putting epicenter and many other cities on lockdown, keeping it from being exported to other countries, and aggressively tracing anyone the infected were in contact with and quarantining those people for 2 weeks [21]. However, the devastating SARS-CoV2 seems unstoppable to march stealthily toward many more places and shows high possibility to engender another pandemic. These facts suggest that measures taken at present such as infection quarantine and travel restrictions may not be adequate to contain the epidemics, especially, the ones caused by the air-borne viruses aggressing host respiratory system. Vaccines are thought to be the ultimate arsenal enabling people to finally control such SARS-like life-threatening epidemics. As such, researchers are racing against time to develop vaccines that should be promptly available in sufficient quantities for massive use [22].

Conventional vaccines using the attenuated live microbes and the inactivated/killed microbes can hardly meet with the requirements of handling urgent epidemics. These kinds of vaccines are manufactured thorough production of large volumes of pathogens involving microbe proliferation and egg/cell culture medium preparation, thus engendering a considerable lag time between vaccine development and vaccination conduct [23]. Novel modes of making vaccines tend to focus on purified antigens (Ags), e.g., using Ag-coding DNA and mRNA to develop a nucleic acid vaccine [24], engineering viruses to deliver whole Ags as a recombinant vaccine, and making expression system to secret Ags for formulating a subunit vaccine [25]. Nucleic acid vaccines can be developed rapidly with the Ag sequence, e.g., an mRNA vaccine against SARS-CoV2 was developed ready for clinical trial in just 6 weeks from the virus sequence identification. But DNA/mRNA vaccines are rigid in formulating with an adjuvant, which is usually indispensable for enhancing immunostimulatory efficacy of Ags presented alone [26]. Preparation of recombinant virus-vectored vaccines also involves lengthy large-scale culturation of microbes and often takes time long enough to miss the vaccination window of an epidemic [27]. By comparison, subunit vaccines using purified Ags rapidly expressed by a microbial system can be flexibly combined with a vaccine adjuvant-delivery system (VADS) to achieve immunization purpose. A VADS can fulfil multiple immunostimulatory functions such as targeting delivery, changing immune response type, and promoting comprehensive cellular as well as humoral reactions [28]. Also, it has been confirmed that mucosal vaccination is most efficient in inducing robust immunity at the administration site [7]. Therefore, various nanoparticulate carriers have been developed as a pulmonary VADS for delivering subunit vaccines but few succeed in taming the highly contagious pathogens, such as SARS and MERS CoVs, which invade hosts via respiratory mucosa [29].

Notably, the most frequently used VADS is alum (aluminum salt) which has been used in many clinical vaccines since 1920s [30]. The alums in licensed vaccines include aluminum hydroxide, aluminum phosphate and potassium aluminum sulfate, all of which can effectively enhance antibody response to vaccines with, however, not-exactly known mechanisms [31]. Whatever aluminum salt is used as an adjuvant, in an aqueous medium, its acid groups will always be substituted to some degree by the anionic hydroxyls [32]. The latter facilitate formation of hydrogen bonds between alum primary nanoparticles, which are thus associated into micrometer clusters engendering a gel-like suspension. The gel-like alum adjuvants may not fit certain administration modes, such as pulmonary vaccination, since their sticky 
property is likely to cause bronchoalveolar airway obstruction. Moreover, alums have another two disadvantages: they are a strong antibody adjuvant but unable to induce cellular reactions; they sometimes stimulate unacceptable local irritations as side effects [33]. As a result of this, researchers are now making efforts on reformulation of conventional alums to improve their adjuvanticity. In particular, it was found that engineering of alums into special nanostructures, with/without certain materials, such as phospholipid engendering the phospholipid bilayer-coated aluminum nanoparticles (PLANs) and poly(acrylic acid) generating nanorods, can remarkably reduce their local irritating activity while rendering them able to enhance cellular immune responses toward the carried Ags [34-39]. These outcomes inspire us to trial the feasibility of using aluminum nanoparticles (ANs) as a pulmonary VADS for delivering vaccines to elicit strong mucosal immunity against air-borne pathogens, such as SARS-CoV2 and MERS, that incline to infringe and disable human respiratory system.

In this primary investigation, aluminum oxide nanoparticles (ANs) were used as a VADS to deliver model Ag of OVA (ovalbumin) via pulmonary administration. We found that ANs facilitated APC (Ag-presenting cell) uptake of vaccines and maturation and rendered vaccines lysosome escape to reduce unwanted degradation of Ags while enhancing MHC-I-Ag presentation. Given to mice via pulmonary administration, ANs safely induced robust humoral as well as cellular immune responses. By contrast, pulmonary administration of micron-sized alum to mice caused serious side effects and even damaged them to death. These results suggest that aluminum oxide NPs may a pulmonary VADS for delivering vaccines to elicit immunity against the respiratory system-invading pathogens, such as SARS-CoV2.

\section{Experimental Details}

\subsection{Materials and Animals}

The following products were purchased from Sigma-Aldrich (Merck, Darmstadt, Germany): the $30 \mathrm{~nm}$-sized ANs (aluminum oxide nanoparticles), calcein (sodium salt), MTT (3-(4,5-dimethylthiazol-2-yl)-2,5-diphenyltetrazolium bromide), DAPI (4',6-diamidino-2-phenylindole), CFSE (5(6)-carboxyfluorescein diacetate $N$-succinimidyl ester), TMB (3,3,5-tetramethylbenzidine), BSA (bovine serum albumin), and OVA (ovalbumin). Goat anti-mouse IgGhorse radish peroxidase (HRP), IgG1-HRP, IgG2a-HRP, and mouse cytokines IFN- $\gamma$ and IL-4 ELISA assay kits, fluorochrome-labeled anti-mouse Abs against different cell surface antigens such as CD40, CD80 and CD86 for APC activation assay, and fluorescent agent-conjugated anti-mouse $\mathrm{CD} 4+\mathrm{mAb}$ and $\mathrm{CD} 8+\mathrm{mAb}$ for $\mathrm{T}$ lymphocytes activation assay were purchased from either eBioscience (San Diego, USA) or BioLegend (San Diego, USA). GM-CSF (granulocyte-macrophage colony stimulating factor), LysoTracker®red, HyClone RPMI 1640, DMEM/F12 cell culture medium, and fetal bovine serum were purchased from Thermo Fisher Scientific Inc. (Waltham, MA, USA). Analytic grade agent of aluminum phosphate $\left(\mathrm{AlPO}_{4}\right)$ and other chemicals were provided by Sinopharm Chemical Reagent Co., Ltd. (Beijing, China). Pure water was produced using a Milli-Q ${ }^{\circledR} \mathrm{IQ}$ 7000 Ultrapure Water System (Merck, Germany).

Female Kunming mice (6-8 weeks) were provided by the Experimental Animal Center of Anhui Medical University (approval number LLSC20190286) and were maintained under specific pathogen-free conditions in the animal facility. All experimental procedures were following the bioethical rules and approved by the Institutional Ethical Committee of Anhui Medical University.

\subsection{Al-Carrier Preparation and Characterization}

ANs $\left(\mathrm{Al}_{2} \mathrm{O}_{3}\right.$ nanoparticles) with a size of around $30 \mathrm{~nm}$ were a commercial product by Sigma-Aldrich. $\mathrm{AMs}\left(\mathrm{AlPO}_{4}\right.$ microparticles) with a size of around $2 \mu \mathrm{m}$ were prepared by mechanical smashing $\mathrm{AlPO}_{4}$ crystal salt in a mortar using a pestle. ANs and AMs were characterized of size and zeta potential by DLS (dynamic light scattering) and ELS (electrophoretic light scattering) using Zetasizer Nano ZS90 (Malvern Panalytical Ltd., Malvern, UK). ANs were further observed of shape and size using a SEM (scanning electron microscope) (JSM-7001F, JEOL (Japan Electron Optics Laboratory) Ltd., Tokyo, Japan). For Ag loading, an aqueous suspension of $\mathrm{Al}$ carriers was mixed with an OVA (model Ag) solution under vigorous stirring making use of phosphophilic property aluminum [38].

\subsection{In Vitro APC Uptake of AI-Carriers and Intracellular Localization of AI-Nanocarriers}

ANs and AMs were characterized of size and zeta potential using Marlven 90-APC (BMDC) uptake of Al-based carriers, including AMs and ANs, which were calcein-labeled (calcein/alum $=1: 90, \mathrm{w} / \mathrm{w}$ ) for observation convenience. BMDCs (mouse bone marrow-derived dendritic cells) were differentiated with GM-CSF (granulocyte-macrophage colony stimulating factor) from mouse bone marrow precursors according to previous reference [40]. BMDCs were seeded in a 24-well plate with $10^{6}$ cells/well and incubated for $24 \mathrm{~h}$ in a cell culture chamber at $37{ }^{\circ} \mathrm{C}$ at an atmosphere of $5 \%$ $\mathrm{CO}_{2}$. Then, the cells were dosed with calcein-labeled Alcarriers $(30 \mu \mathrm{g} \mathrm{Al} / \mathrm{ml})$ for another $4 \mathrm{~h}$ incubation in the cell culture chamber and, after thrice washing with PBS, were collected for flow cytometry and other analysis. 
For laser scanning confocal microscope (LSCM, Leica TCS SP5, and Wetzlar, Germany) observation, the diluted cells were stained of lysosomes for $1 \mathrm{~h}$ using LysoTracker®red. Then cells were fixed in an aqueous $4 \%$ formaldehyde solution for $15 \mathrm{~min}$ and stained of nuclear with DAPI for $10 \mathrm{~min}$. After thrice washing with PBS, the cells were visualized by LSCM.

\subsection{APC Maturation In Vitro and Cytotoxicity of Al-Based Nanocarriers}

The cytotoxicity of Al-based carriers was analyzed with an MTT assay using RAW264.7 macrophages, which were seeded in a 96-well plate at a concentration of $10^{5}$ cells/ well. Following $24 \mathrm{~h}$-incubation in a cell culture chamber, the cells were co-cultured with various concentrations of $\mathrm{Al}$ carriers for $24 \mathrm{~h}$. After removing $\mathrm{Al}$-particles and culture medium and thrice PBS washing, cells were supplemented with $0.5 \mathrm{mg} / \mathrm{ml} \mathrm{MTT-containing} \mathrm{cell-culture} \mathrm{medium} \mathrm{for}$ another $4 \mathrm{~h}$-incubation at $37^{\circ} \mathrm{C}$. After removal of supernatant, each well was added with $100 \mu \mathrm{l}$ DMSO to fully dissolve the MTT-induced crystals within cells and detected of the optical absorbance at $490 \mathrm{~nm}$ with a $\mu$ Quant microplate reader (Bio-Tek, Winooski, VT, USA).

BMDCs were incubated in a 24-hole plate $\left(10^{6}\right.$ cells/well $)$ together with PBS, OVA, OVA-ANs, OVA-AMs, at a dose of $20 \mu \mathrm{g}$ OVA for $30 \mathrm{~h}$. Afterward, the cells were washed three times with PBS and were specifically bound with the fluorochrome-conjugated Abs against CD40, CD80 and CD86, respectively. Then, each group was analyzed by flow cytometry for evaluation of cell maturation.

\subsection{Animal Immunization}

Different groups of mice $(n=6)$ were anesthetized by intraperitoneal injection of chloral hydrate at a dose of $0.5 \mathrm{mg} / \mathrm{g}$ body weight and then immunized by pulmonary administration of saline, OVA in saline, OVA-ANs $(1: 10, \mathrm{w} / \mathrm{w})$, or OVA-ANs $(1: 20, w / w)$ at the dose of $10 \mu \mathrm{g} / 10 \mu \mathrm{l}$ of OVA. One group of mice was immunized subcutaneously at the lateral aspect of the left thigh near the knee with OVA-AMs $(1: 20, \mathrm{w} / \mathrm{w})$ at the same OVA dose as a positive control. The pulmonary administration was performed by a simple method for endotracheal intubation of mice as described in literature [41]. All the groups of mice were immunized twice a 3-week interval.

\subsection{Sample Collection and Antibody Determination}

The biological samples from the immunized mice were collected 3 weeks after the second vaccination under anesthesia. The blood was collected from the retro-orbital plexus and allowed to stand at room temperature for $20 \mathrm{~min}$ and then centrifuged for $10 \mathrm{~min}$ at $10,000 \mathrm{rpm}$ for collecting the supernatant serum, which was then stored at $-20^{\circ} \mathrm{C}$ until further assay.

Mouse fecal samples were obtained by collecting overnight feces from each group one day before blood sampling. After pulverization and mixing, $2 \mathrm{~g}$ of feces from each group was suspended in $2 \mathrm{ml}$ PBS and vortexed for $5 \mathrm{~min}$. The sample suspensions were filtered with filter paper, and the filtrate was centrifuged at $16,000 \times g$ for $10 \mathrm{~min}$ to collect supernatants, which was stored at $-20^{\circ} \mathrm{C}$ for further assay.

The vaginal flush samples were collected by two successive washes with $100 \mu$ l of PBS, which was repeated introduced into and withdraw from the vaginal tract for 10 times using a pipette, and the two washes were pooled. In the similar way, oral and nasal cavity flush samples were collected from mice by twice washing of oral and nasal cavities with 400 and $1000 \mu \mathrm{l}$ of PBS, respectively.

Small intestinal flush and BALF (broncho-alveolar lavage fluid) samples were obtained through twice washing of the isolated small intestines and lungs with 1 and $4 \mathrm{ml}$ of PBS, respectively.

All mucosal flush samples were clarified by centrifugation at 10,000 rpm for $10 \mathrm{~min}$ to collect the supernatants, which were stored at $-20{ }^{\circ} \mathrm{C}$ until further assay.

The antibody tests were carried out by ELISA protocol according to a previous report [38]. In addition, the sera from immunized mice were diluted with PBS by 1:5000 for IgG assay and 1:200 for IgG1 and IgG2a assay. For IgA assay, the feces extraction solutions were diluted by 1:500, while the cavity mucosa-rinsing fluids were diluted by 1:50.

\subsection{Splenocyte Proliferation, Subset and Cytokine Assay}

Three weeks after immunization, mice were anesthetized and, with aseptic operation procedures, were isolated of splenocytes.

For splenocyte proliferation assay, the isolated cells were suspended in a complete cell growth medium consisting of DMEM, $0.1 \mathrm{mg} / \mathrm{ml}$ penicillin and streptomycin and $10 \%$ (v/v) fetal bovine serum. And $100 \mu \mathrm{l}$ of $5 \times 10^{5}$ cells were seeded in each well of a 96-well plate and incubated in the presence of $1 \mathrm{mg} / \mathrm{ml} \mathrm{OVA}$ for $72 \mathrm{~h}$ at $37^{\circ} \mathrm{C}$ in a cell chamber containing $5 \% \mathrm{CO}_{2}$. Thereafter, the plate was centrifuged at $1000 \mathrm{~g}$ for $10 \mathrm{~min}$ to collect the supernatants which were then stored at $-20{ }^{\circ} \mathrm{C}$ for further assay of cytokines such as IFN- $\gamma$ and IL-4. And then cells were washed 3 times with PBS for MTT assay, whereby the optical absorbance (OA) at $490 \mathrm{~nm}$ wavelength of each well of the plate was measured using $\mu$ Quant microplate reader. Then, the stimulation index (SI) representing cell proliferation was expressed as equation: $\mathrm{SI}=\mathrm{OA}$ of experimental cells/OA of control cells. 
For cytokine tests, a sandwich enzyme immunoassay was used to determine IFN- $\gamma$ and IL- 4 in mouse sera or in the culture supernatants of the $72 \mathrm{~h}$ OVA-re-stimulated splenocytes.

For T lymphocyte subset assay, the isolated splenocytes were suspended in PBS with a concentration of $10^{5}$ cells $/ \mathrm{ml}$. Then cells were stained with $0.5 \mu \mathrm{g}$ of APC-conjugated antimouse $\mathrm{CD} 4 \mathrm{mAb}$ and FITC-conjugated anti-mouse CD8 $\mathrm{mAb}$ at $4{ }^{\circ} \mathrm{C}$ for $2 \mathrm{~h}$ in the dark. Then after centrifugation at $1000 \mathrm{~g}$ for $5 \mathrm{~min}$ and two washes with PBS, the cells were resuspended in PBS in a tube and assayed by flow cytometry.

\subsection{Histopathology Assay for In Vivo Safety Evaluation}

Vaccine-associated inflammation at the lung was evaluated by histological section analysis. Mice $(n=3)$ were given with normal saline, OVA-ANs $(1: 20, \mathrm{w} / \mathrm{w})$, or OVA-AMs $(1: 20, \mathrm{w} / \mathrm{w})$ at the dose of $10 \mu \mathrm{g} / 10 \mu \mathrm{l}$ OVA by pulmonary administration and were then observed of the activity state. Three days later, pieces of lung tissues were isolated fixed in $4 \%$ paraformaldehyde solution $(24 \mathrm{~h})$, dehydrated in ethanol, cleared in toluene and embedded in paraffin wax. Lung tissue sections with 5- $\mu \mathrm{m}$ thick were cut using a wax slicing machine (RM2255, Leica Biosystem, Germany) and stained with hematoxylin and eosin (H\&E). Finally, the lung tissue histological sections were analyzed of inflammatory reactions by light microscopy (Pannoramic SCAN II, 3DHISTECH, Budapest, Hungary).

\subsection{Statistical Analysis}

Results were presented as mean \pm SD (standard deviation). Statistical differences between experimental and control groups were estimated by unpaired Student's $t$ test with the SPSS software. A p value $<0.05$ was considered to be significant.

\section{Results}

\subsection{Characteristics of Lipids Adsorbed on Al-Based Nanocarriers}

The size and morphology of the ANs were observed in the scanning electron microscope (SEM) (Fig. 1), and SEM image showed that ANs loaded with OVA have a size of around $40 \mathrm{~nm}$. The mean diameter, zeta potential ( $\zeta$ ), OVA association efficiency (AE) of ANs, OVA-loaded ANs are presented in Table 1 . The size and $\zeta$ of both ANs and OVAANs were of around $25 \mathrm{~nm}$ and $-30 \mathrm{mV}$, respectively, and changed little before and after OVA loading, which had an AE of above $80 \%$.

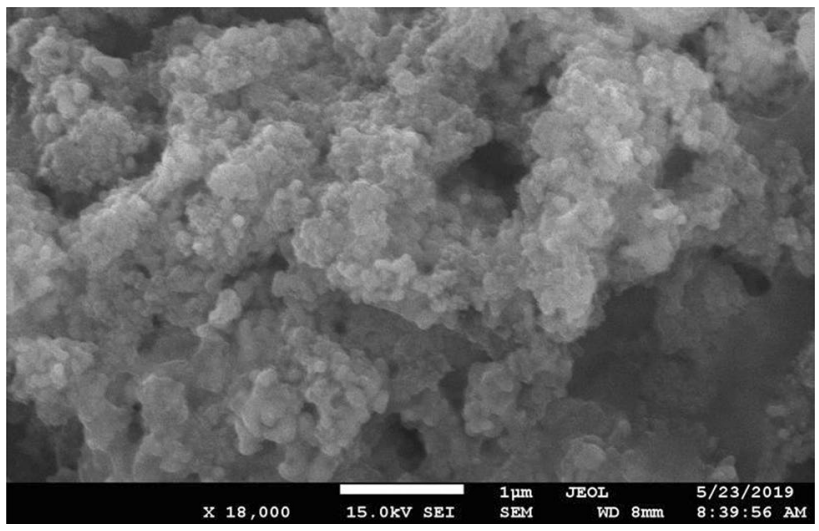

Fig. 1 SEM image showing the morphology and size of the OVAloaded ANs

Table 1 The size (mean \pm SD) and distribution (PDI) (mean \pm SD), zeta potential (mean $\pm \mathrm{SD}$ ) and association efficiency (AE) for OVA of the ANs, PLANs, and lung-PLAN $(\mathrm{n}=3)$

\begin{tabular}{lllll}
\hline sample & $\begin{array}{l}\text { Size } \pm \text { SD } \\
(\mathrm{nm})\end{array}$ & PDI \pm SD & $\begin{array}{l}\text { Zeta } \pm \text { SD } \\
(\mathrm{mV})\end{array}$ & AE \pm SD $(\%)$ \\
\hline AN & $24.86 \pm 4.4$ & $0.39 \pm 0.01$ & $-34.2 \pm 3.3$ & - \\
OVA-AN & $28.3 \pm 0.57$ & $0.45 \pm 0.03$ & $-27.7 \pm 0.2$ & $83.8 \pm 1.8$ \\
\hline
\end{tabular}

The release of OVA from ANs was rather slow, and after $48 \mathrm{~h}$ incubation in either PBS or simulated lung fluid at $37{ }^{\circ} \mathrm{C}$, only about $40 \%$ of total $\mathrm{Ag}$ were freed into medium, in agreement with our previous investigation [38].

\subsection{In Vitro AI-Nanocarriers Enhancing APC Uptake and Safety of Alum}

Calcein, a membrane-impermeable fluorescent agent, was loaded by Al-carriers to investigate APC uptake of the carrier delivered cargoes. As shown in the LCSM (laser confocal scanning microscopy) fluorescent images (Fig. 2a), ANs significantly facilitated cellular uptake of cargoes by BMDCs to contrast AMs, which were rarely associated with the co-incubated cells. To confirm these results, the BMDCs co-incubated with Al-carriers were further subjected to flow cytometry assay. The quantitative analysis results demonstrated that the fraction of the BMDCs had taken up calceinANs was remarkably higher than that had taken up calceinAMs or free calcein (Fig. 2b).

The safety of ANs was evaluated by MTT tests, of which the outcomes indicated that ANs had little influence on viability of macrophages (Raw264.7) (Fig. 2c). By contrast, AMs ( $\mathrm{AlPO}_{4}$ microparticles) significantly reduced cell viability when at concentration higher than $50 \mu \mathrm{g} / \mathrm{ml}$. 


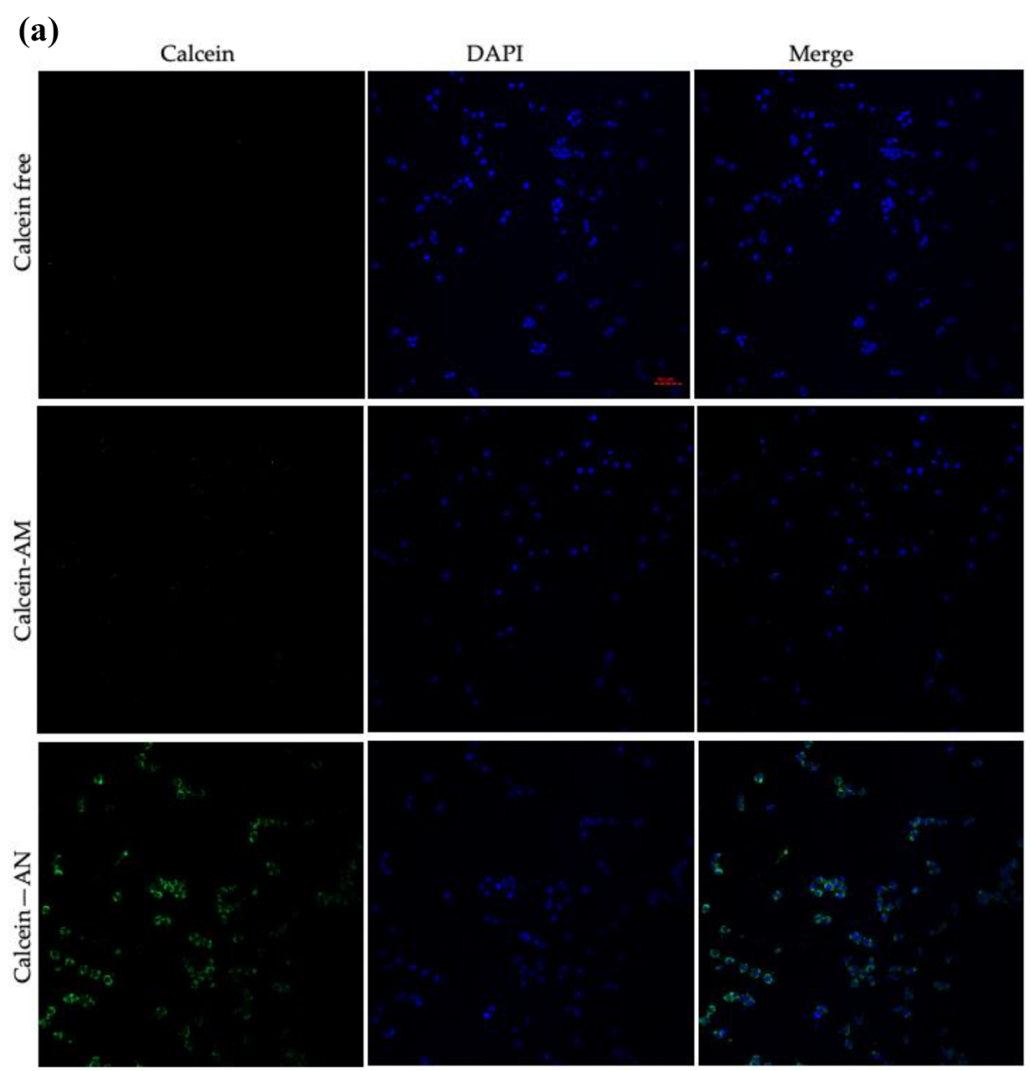

(b)
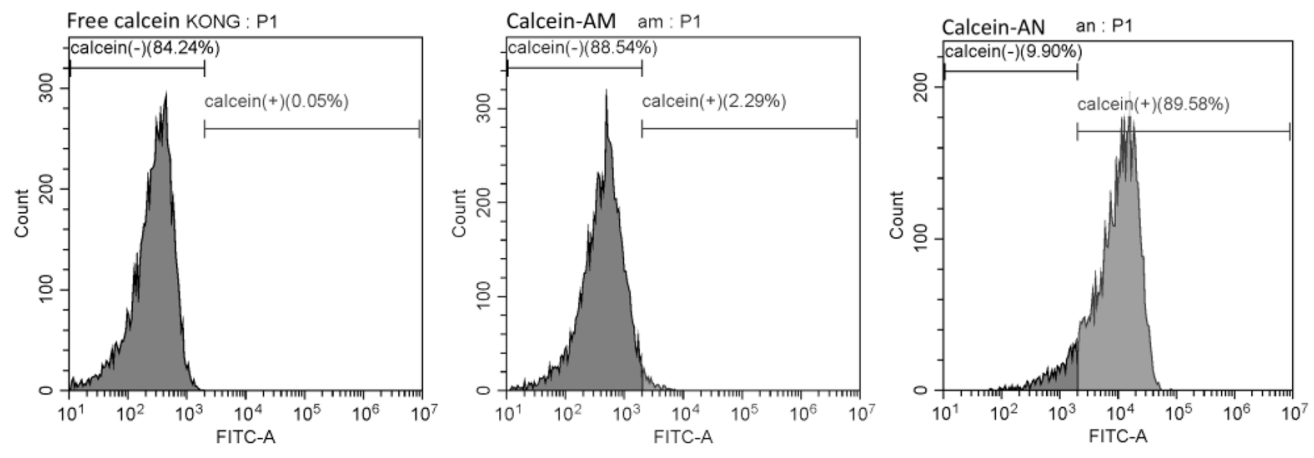

(c)

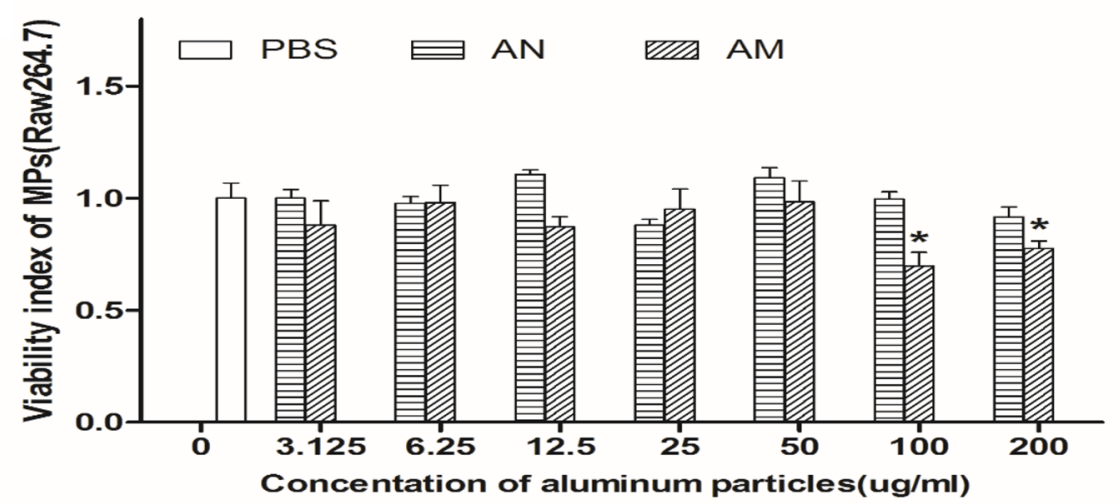


4Fig. 2 Cellular uptake and toxicity profiles of the Al-based carriers $(n=3)$. a CLSM fluorescent images showing mouse BMDC uptake of free calcein, calcein-ANs or -AMs (bar $=50 \mu \mathrm{m}$, applicable to all panels). $\mathbf{b}$ The percentages of APCs that had internalized calcein free in saline or associated with Al-carriers, with the data obtained by flow cytometry. c APC viability index derived from the MTT analysis obtained through incubation of macrophages (Raw264.7) with PBS, ANs or AMs

\subsection{APC Activation by Ag-Loaded Al-Nanoparticles Facilitating Lysosome Escape}

APC activation by Ag-loaded Al-carriers was also evaluated through flow cytometry assay of expression of CD40, CD80 (also called B7-1) and CD86 (also called B7-2) on BMDCs. These molecules are argued as APC activation markers, as they have been identified to involve in ligating receptors on $\mathrm{T}$ cells, i.e., CD40-to-CD40L and CD80/CD86-to-CD28, providing costimulatory signals necessary for $\mathrm{T}$ cell activation and survival $[42,43]$. As shown in (Fig. 3a), while free OVA exerted little stimulation effects on APCs (BMDCs), OVAAMs only triggered APCs to express CD40 and CD86 at a slightly elevated level. By contrast, OVA-ANs significantly activated BMDCs and remarkably elevated their expression of all the three marker molecules CD40, CD80, and CD86.

To clarify whether ANs have the ability to deliver Ags while getting around of unwanted lysosomal degradation, intracellular tracking of ANs was performed through using LSCM to image the calcein-labeled ANs and the LysoTracker ${ }^{\circledR}$-red-stained lysosomes. The fluorescent images (Fig. 3b) demonstrated that the ANs endocytosed by APCs located at sites other than lysosomes, suggesting the Al-nanocarriers have the ability to render the delivered Ags lysosomal escape.

\subsection{Local Inflammations Caused by Al-Carriers Administered via Pulmonary Route}

Safety of pulmonary administration of Al-carriers was evaluated by histopathological analysis of the lung tissue sections stained with H\&E. The microscopic images indicated that, like normal ones (Fig. 4a), the lung issues of mice received ANs (Fig. 4b) showed no sign of significant histopathological alterations, though they were noticed of a few of cells the tissue areas close to certain blood vessels. By contrast, pulmonary administration of AMs caused a plethora of inflammatory cells to infiltrate mouse whole lung tissues (Fig. 4c). In addition, death as well as body-weight loss that occurred in mice received AMs completely denied alum to act as a pulmonary VADS (data are not shown). Similar damages were also observed in mice intranasally immunized with alum adjuvanted RSV (respiratory syncytial virus) vaccines by other researchers [44]. Nevertheless, outcomes of this investigation proved that nanotechnology still works well in improving adjuvanticity of alums, so that it allows $\mathrm{Al}_{2} \mathrm{O}_{3} \mathrm{NPs}$ to be used as a VADS for pulmonary vaccination.

\subsection{Ag-Specific Immune Responses Induced by Al-Nanocarriers}

OVA-ANs were also tested in mice of the ability to evoke the immune responses toward Ags via pulmonary administration. Because pulmonary vaccination of AMs was noticed of severe cytotoxicity, mice in the alum control group were immunized by subcutaneous injection (s.c.). All the animals were immunized twice with a 3-week interval. ELISA examination revealed that, by pulmonary administration, OVA-ANs were much more efficient than free OVA in inducing synthesis of anti-Ag IgG (Fig. 5a). Notably, at a high AN dose (OVA/ AN of 1:20), the serum IgG levels in OVA-AN mice even surpassed the levels in s.c. OVA-AM mice after first vaccination but declined significantly after the second vaccination. A similar trend was also observed in vaccine-induced anti-Ag IgG1 and IgG2a, both of which showed lowered levels after second mouse vaccination in either OVA-AN or OVA-AM group (Fig. $5 \mathrm{~b}$ and c). The exact reason for this remains to be fully disclosed but might well be argued relevant to certain side effects associated with pulmonary ANs or s.c. AMs, as mouse bodyweight in either vaccine group seemed to decrease after first immunization (Fig. 5d). IgG1 and IgG2a isotypes are argued as markers for Th2 and Th1 lymphocytes, respectively, and thus may present some insight into the type of Th cell immune response [45]. IgG2a-to-IgG1 ratio values in mice received OVA-ANs were much higher than that received free OVA or OVA-AMs, suggesting Al-nanocarriers have a tendency to induce a more balanced $\mathrm{Th} 1 / \mathrm{Th} 2$ response. This observation was further supported by cytokine assay outcomes, which indicated that mice stimulated by pulmonary OVA-ANs produced more IFN- $\gamma$, whereas those triggered by s.c. AMs secreted more IL-4 (Fig. 5e and f).

Additionally, pulmonary vaccination with AN-delivered OVA triggered mice to generate widespread and robust mucosal immune responses, as revealed by presence of $\operatorname{Ig} \mathrm{A}$ in saliva, nasal and vaginal washes, and, especially, BALF (Fig. 5g). Although the second pulmonary OVA stimulation also induced mouse production of $\operatorname{IgA}$, this kind of mucosal reaction was limited to respiratory system, i.e., the local sites subjected to vaccination. Unexpectedly, mice receiving twice s.c. OVA-AMs were also evoked to make $\operatorname{IgA}$ in saliva, nasal and vaginal washes, as well as BALF, but to a lesser degree compared to that of OVA-AN group.

\subsection{Differentiation and Proliferation of Splenocytes from Vaccinated Mice}

Also, flow cytometry assay of the splenocytes of vaccinated mice indicated that the Al-based carriers induced production 
(a)
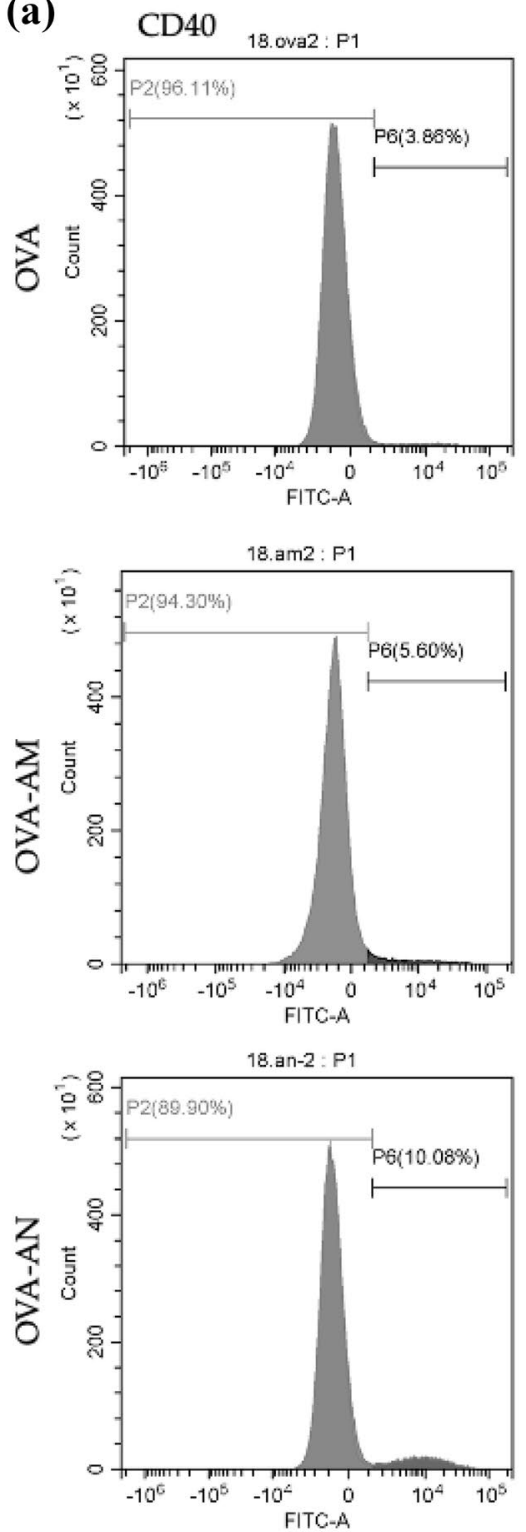
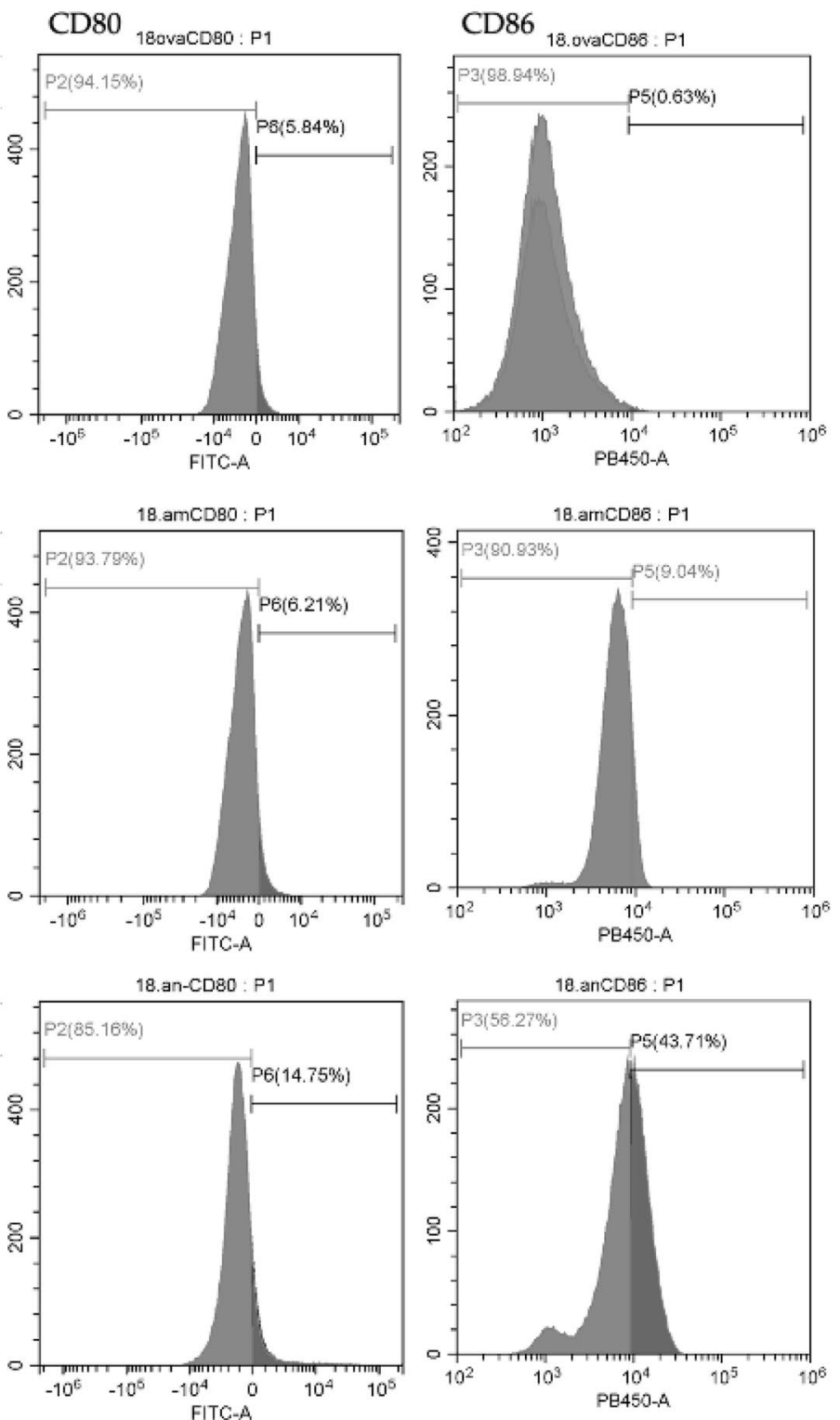

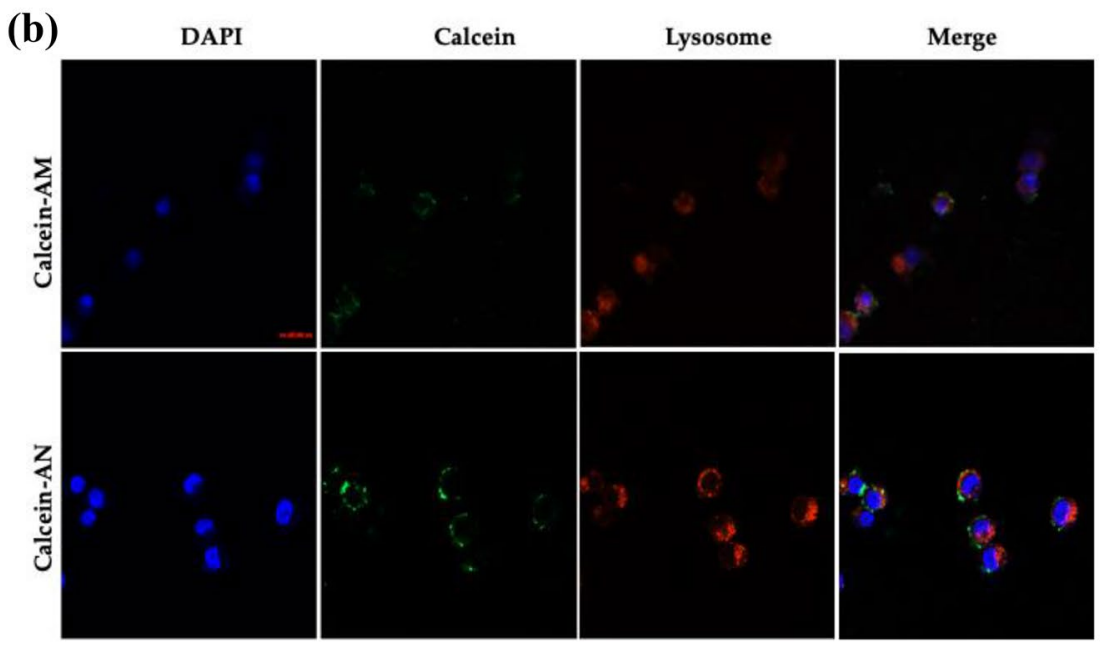


4Fig. 3 a The expression levels of activation biomarkers (CD40, CD80 and CD86) on APCs after stimulation with free OVA, OVA-AMs, or OVA-ANs. Data were obtained by flow cytometry assay of BMDCs coincubated with different formulations for $30 \mathrm{~h}$ and then stained with fluorescent-labeled anti-CD Abs. b LSCM image for localization of intracellular calcein-loaded ANs endocytosed by APCs which were stained with DAPI for labeling nucleus (blue) and with LysoTrack-red for labeling lysosomes (red) ( $\mathrm{bar}=10 \mu \mathrm{m}$, applicable to all panels) (Color figure online)

of much higher frequency of $\mathrm{CD} 8^{+}$lymphocytes than did the micro-sized alum, suggesting the Al-based nanocarriers as a VADS having the potential to elicit cellular immunity.

The splenocyte proliferation in mice immunized with Albased nanocarriers was tested with the MTT method. As shown in Fig. 6, regardless of the immunization route, both OVA-AN and OVA-AM formulations remarkably promoted splenocyte proliferation compared to the negative control.

\section{Discussion}

This investigation proved that ANs performed well as a pulmonary VADS, which not only induced robust humoral and cellular immunity at systemic as well as mucosal levels, but also exhibited a high safety profile. By contrast, gel-like alums exist in micron-sized clusters and are known, and evidenced again in this report, able to promote only humoral reactions besides the infeasibility of pulmonary immunization due to its sticky property and local irritations [31, 32]. The sticky property is apparently resulted from the gels aggregated at administration site and aggravated by secreted mucus leading, possibly, to pulmonary obstruction. Local irritation may arise from actions and disturbance exerted by bulk alum on the contacted cell membranes [46]. These effects may also present high stress and even damages to cells, thus aborting cellular uptake and immune responses while causing irritating inflammation instead (Figs. 2 and 3b) $[28,31]$.

Contrarily, ANs can be administered to the lung and approach individually the site of alveola to trigger APCs, perhaps mainly, AMPs (alveolar macrophages), which instruct the related cells such as DCs and AECs (alveolar epithelial cells) into an active state to make an immune response [47]. Pulmonary vaccination favors setup of mucosal immunity across most of human organs, especially, the respiratory system and should be particularly beneficial
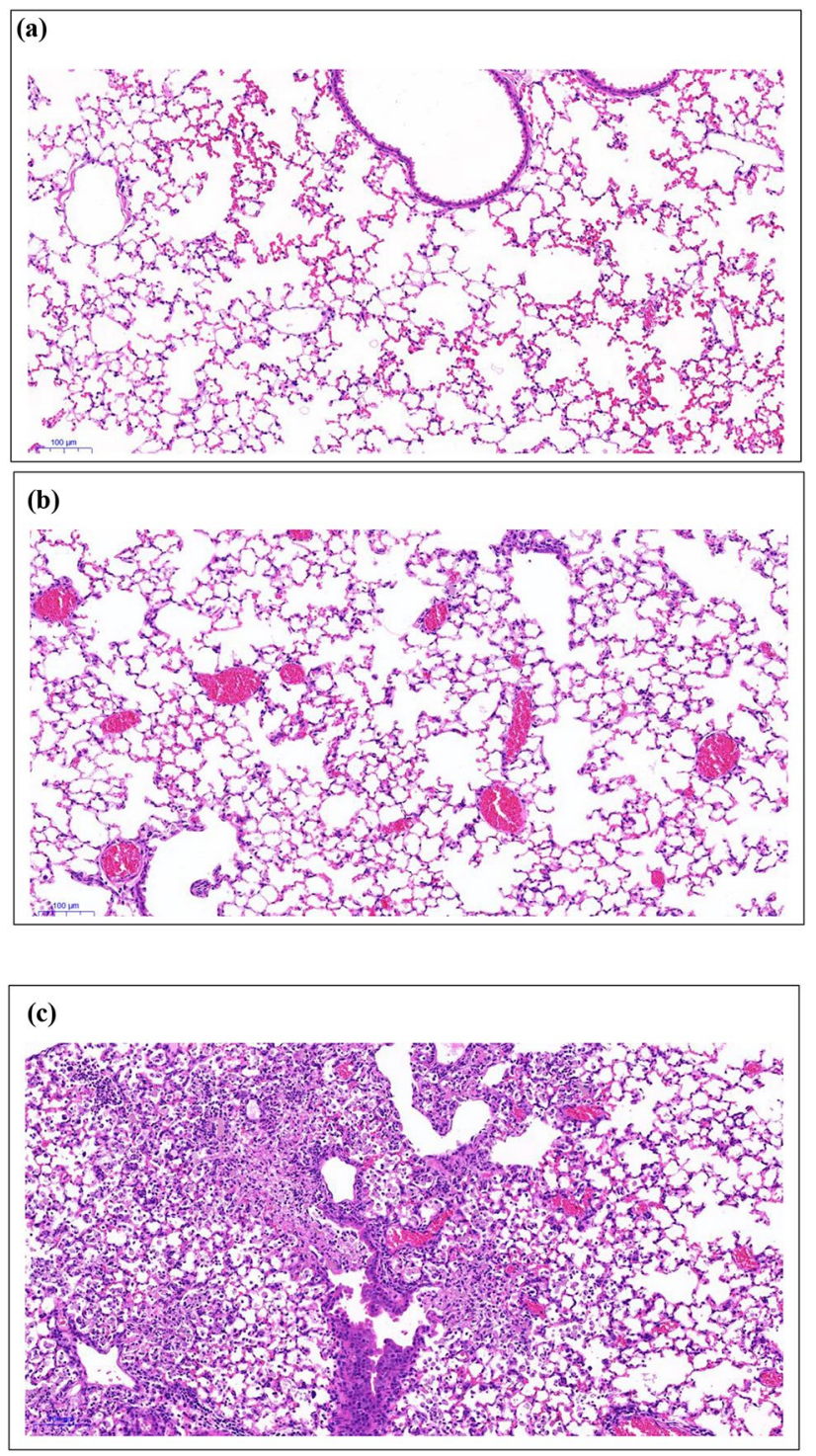

Fig. 4 Histopathological images of the lung tissue sections fixed in paraffin and stained with $H \& E$. The lung tissue sections were obtained $72 \mathrm{~h}$ after pulmonary administration of saline (a), ANs (b) or AMs (c). (scale bar, $100 \mu \mathrm{m})(\mathrm{n}=3)$

for defending against the airborne pathogens, such as flu, MERS and SARS-CoV2, which is now just rapidly spreading throughout the world [48]. The tendency of ANs to facilitate cellular immunity observed in this report was also specifically described in a previous study, wherein alum was engineered as nanosized rods loaded with the recombinant 
(a)

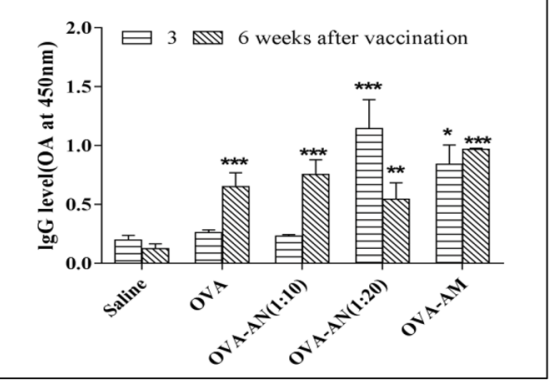

(c)

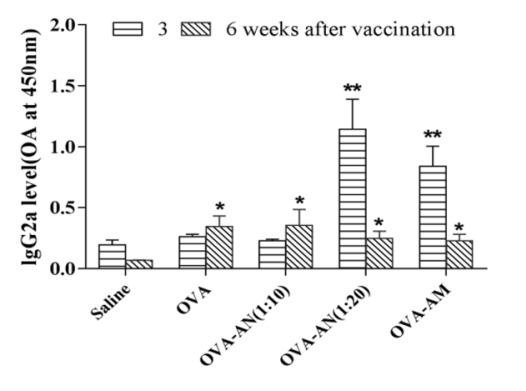

(e)

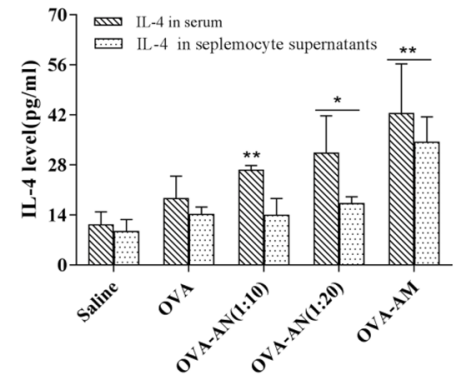

(b)

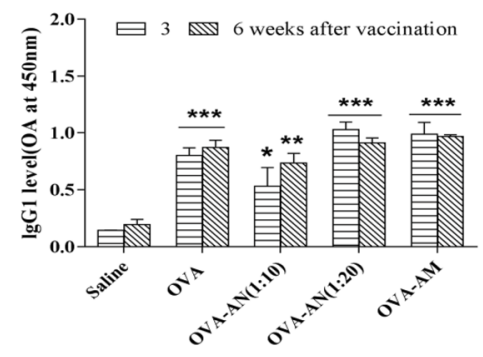

(d)

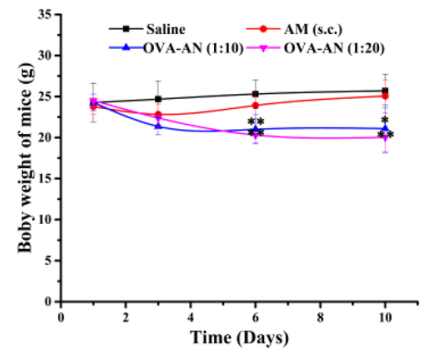

(f)

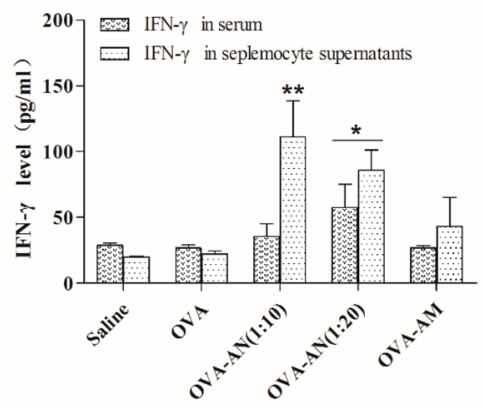

(g)

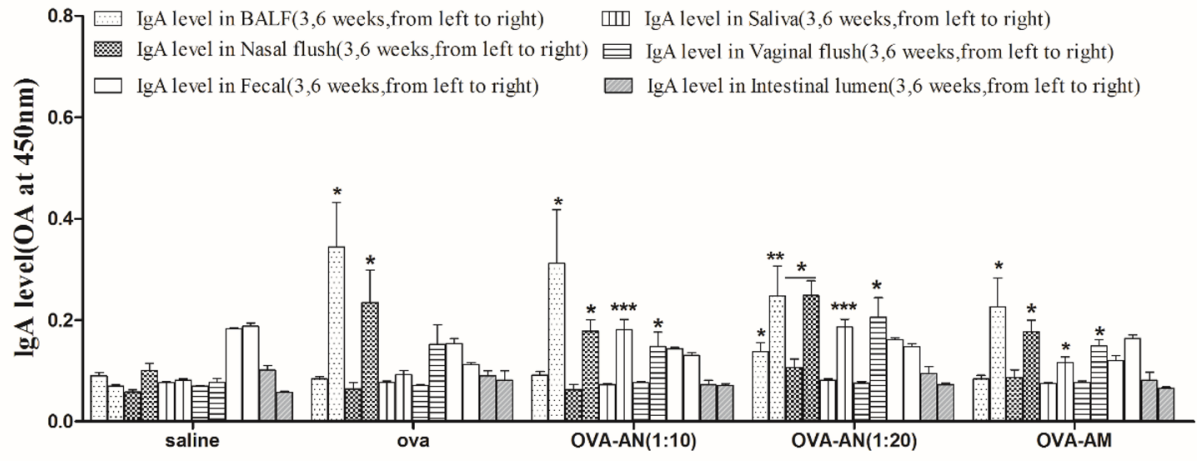


४Fig. 5 Immunoglobulin and cytokine assay of immunized mice $(n=6)$. (A) Levels of IgG in mouse sera (1:5000 dilution) detected after twice vaccinations. b, c Levels of IgG1 and $\operatorname{IgG} 2 \mathrm{a}$ in mouse sera (1:200 dilution) detected after twice vaccinations. (D) Mouse bodyweight tested for ten days after first immunization. (E and F) Levels of IL-4 and IFN- $\gamma$ in mouse sera (1:10 dilution) and splenocyte $\left(1 \times 10^{5}\right)$ cultures. $(\mathrm{G})$ Levels of IgA in different mucosal tissues examined 3 weeks after first and second immunization of mice. (*p value $<0.05, * *$ p value $<0.01$, in comparison to negative control)

tuberculosis Ags [36]. These outcomes support a correlation between the alum size and immunological response [49], although the underlying mechanism remains still elusive but may well be argued here to function as a resulted of APC internalization of the VADS and Ag lysosomal escape (Fig. 3b). Also, minimizing alum into nanoparticles remarkably enhances the VADS traffic to the draining lymph nodes, wherein different types of functional cells have an enlarged chance to be stimulated by vaccines to orchestrate to improve immunological reactions contributing to elevated immunity [14, 37, 38].

The growing shift to subunit antigen vaccines underscores the significance of this AN-based VADS which proves able to enhance the magnitude as well as quality of immune reactions. In particular, this AN-based VADS effectively turns the adjuvanticity of the first VADS, alum, which has a long history of clinical use but predominantly induces Th2 (T helper 2) type immunity characterized by antibody production and lack of antigen-specific CTLs (cytotoxic T lymphocytes) $[30,31,50]$. Notably, the lack of cell-mediated or Th1 immunity makes vaccines ineffective in mounting effective responses against diseases like tuberculosis, malaria, HIV and tumor [51]. Moreover, the highly contagious and dangerous pathogens such as the present rampant SARS-CoV2 may asymptomatically infect a plethora of humans [52]. The asymptomatic virus carriers become stealth resources enormeously facilitating pathogen transmission, which can be interrupted depending, to a large extent, on the availability of the Th1 reaction-inducing vaccines [51].

It is well known that the trachea and bronchi show the mucus-covered epithelial mucosa, however, the alveoli surfaces are mainly coated with a lipid-rich surfactant that has bacteriostatic effects against certain bacterial species [53]. The pulmonary surfactant (PSF) layer is reported to pose a challenge to delivering vaccines to lung APCs by a nanoparticulate VADS vaccines, because the PSF generates a strong barrier to not only pathogen but also nanoparticle accessing mucosa [47, 54]. However, PSF was also described to possess potent adjuvanticities and has been employed as a VADS for enhancing APC uptake and presentation of vaccines $[47,55]$. The influence of PSF on the immunostimulatory activity of AN-based pulmonary VADS remains now unclear and deserves obviously a deepening exploration.

\section{Conclusions}

This investigation demonstrated that engineering micronsized alum into nanoparticles can regulate and improve the adjuvanticity of this conventional vaccine adjuvant. Compared with coarse alum adjuvant, ANs showed enhanced biocompatibility and safety and, especially, the ability for delivering antigens to pulmonary immune cells. Moreover, ANs possessed the adjuvanticity of helping model antigens induce both humoral and cellular immune responses at systemic as well as mucosal level via pulmonary administration. Therefore, ANs may be a promising mucosal VADS for delivering vaccines against pathogens, particularly, the ones that invade hosts via respiratory system, such as MERS and SARS coronaviruses. 


\section{(a) Saline}

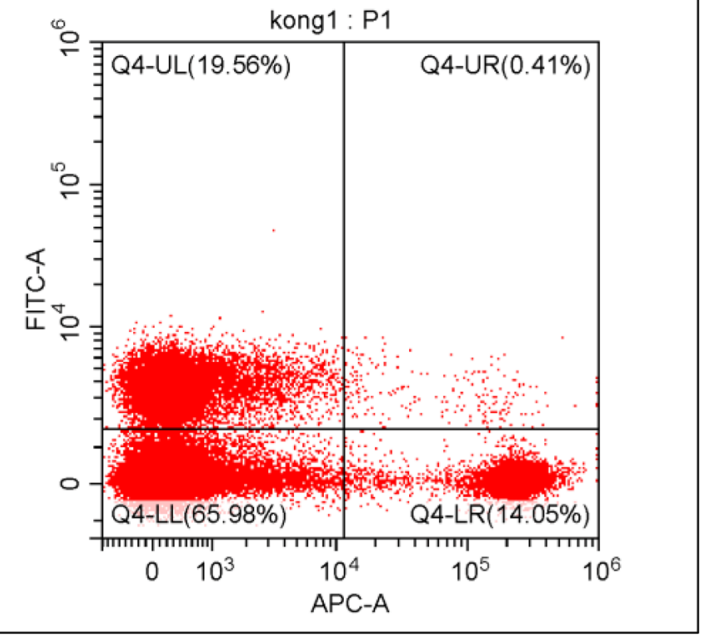

(c) OVA-AN (1:10)

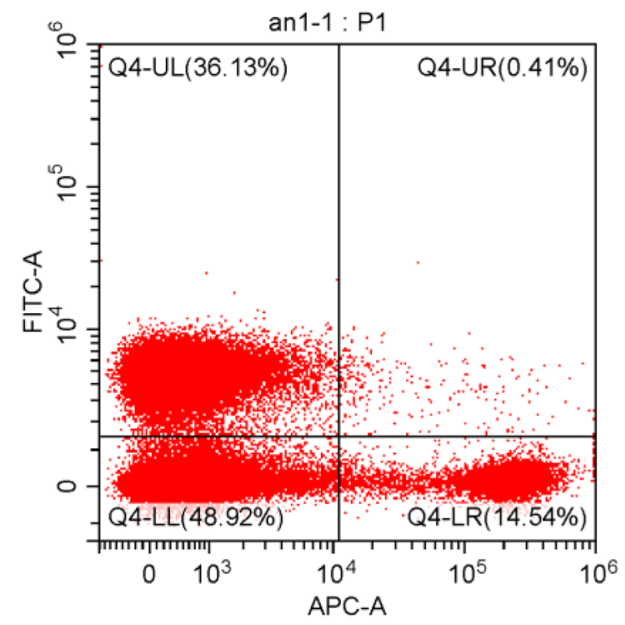

\section{(e) OVA-AM}

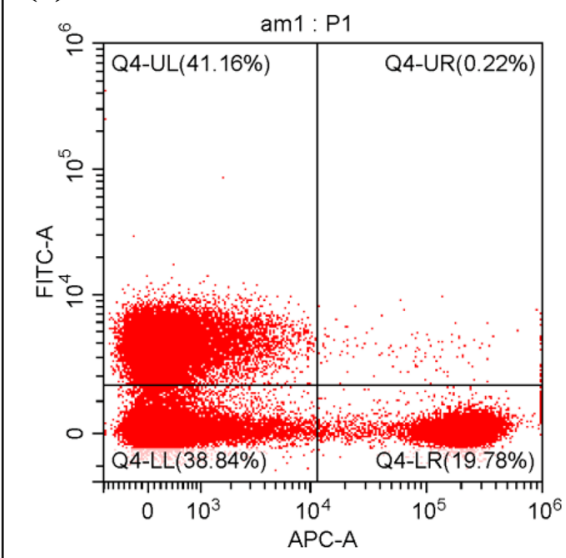

(b) OVA

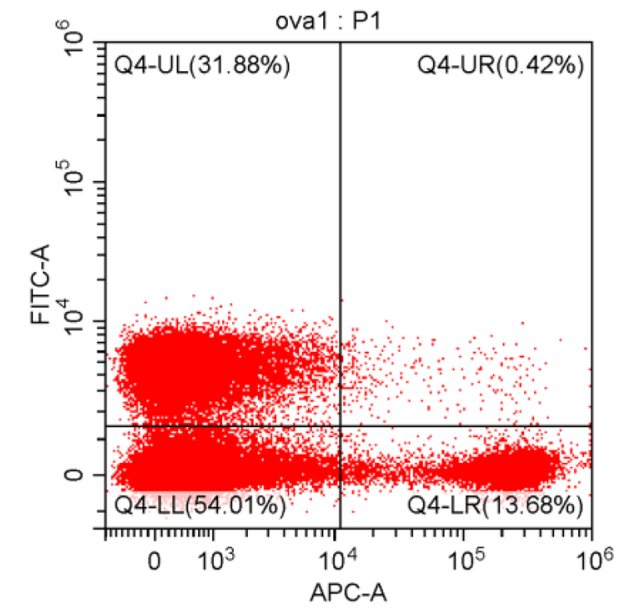

(d) OVA-AN (1:20)

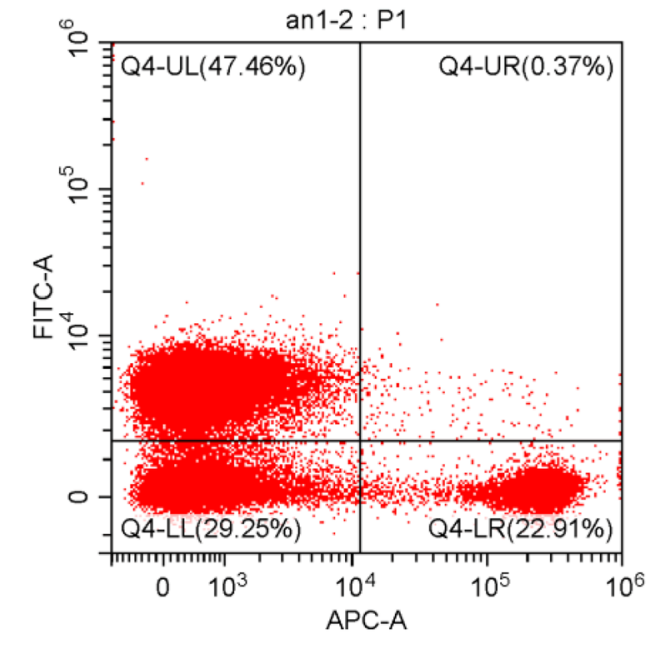

(f)

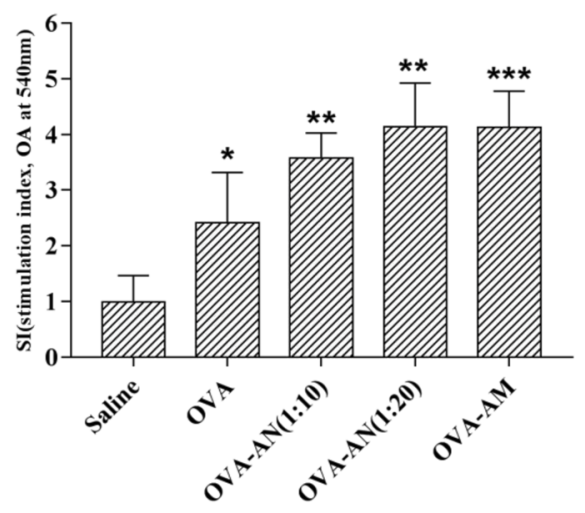


४Fig. 6 Flow cytometry assay of differentiation and proliferation of splenocytes from vaccinated mice $(n=3)$. a-e $\left(1 \times 10^{5}\right)$ labeled with APC-anti-CD8 ${ }^{+} \mathrm{mAb}$ and FITC-anti-CD4 ${ }^{+} \mathrm{mAb}(\mathrm{n}=6)$. f Stimulation index (SI) for mouse splenocyte proliferation by different formulations (*p value $<0.05, * * \mathrm{p}$ value $<0.01$, in comparison to negative control)

Acknowledgements The authors thank Professor Aiping Zheng from Academy of Military Medical Sciences for providing some materials and technique help used for electron microscopy experiments.

Author Contributions The authors state that the author contributions to this work are listed as follows: conceptualization, methodology and supervision, NW and TW; experimental investigation, software and data analysis, CW, ZZ, CW, MC, TL and RQ; data validation and curation, TW and NW; writing and editing, NW, CW and TW; resources, funding acquisition and project administration, NW and TW. Authorship of this report is limited to those who have contributed substantially to the work.

Funding This research was funded by National Natural Science Foundation of China (Grant Number 81703449), Anhui Provincial Natural Science Foundation (Grant Number 1708085QH196) and also the Human Resource \& Social Security Department of Anhui Province (Grant Number 2018H173).

\section{Compliance with Ethical Standards}

Conflict of interest The authors declare no conflict of interest.

\section{References}

1. P. Piot, H.J. Larson, K.L. O'Brien, J. N'kengasong, E. Ng, S. Sow, B. Kampmann, Immunization: vital progress, unfinished agenda. Nature 575, 119-129 (2019). https://doi.org/10.1038/ s41586-019-1656-7

2. S.A. Plotkin, Vaccines: past, present and future. Nat. Med. 11, S5-S11 (2005). https://doi.org/10.1038/nm1209

3. S. Plotkin, History of vaccination. Proc. Natl. Acad. Sci. U S A. 111, 12283-12287 (2014). https://doi.org/10.1073/pnas.14004 72111

4. J.A. Keith, L. Agostini Bigger, P.A. Arthur, E. Maes, R. Daems, Delivering the promise of the decade of vaccines: opportunities and challenges in the development of high quality new vaccines. Vaccine. 31, B184-B193 (2013). https://doi.org/10.1016/j.vacci ne.2012.12.032

5. M.R. Neutra, P.A. Kozlowski, Mucosal vaccines: the promise and the challenge. Nat. Rev. Immunol. 6, 148-158 (2006). https://doi. org/10.1038/nri1777

6. M.M. Levine, "IDEAL" vaccines for resource poor settings. Vaccine. 29, D116-D125 (2011). https://doi.org/10.1016/j.vacci ne.2011.11.090

7. N. Lycke, Recent progress in mucosal vaccine development: potential and limitations. Nat. Rev. Immunol. 12, 592-605 (2012). https://doi.org/10.1038/nri3251

8. A. Lamichhane, T. Azegami, H. Kiyono, The mucosal immune system for vaccine development. Vaccine. 32, 6711-6723 (2014). https://doi.org/10.1016/j.vaccine.2014.08.089

9. Y. Fan, P. Sahdev, L.J. Ochyl, J.J. Akerberg, J.J. Moon, Cationic liposome-hyaluronic acid hybrid nanoparticles for intranasal vaccination with subunit antigens. J. Control. Release. 208, 121129 (2015). https://doi.org/10.1016/j.jconrel.2015.04.010

10. W.F. Tonnis, A.J. Lexmond, H.W. Frijlink, A.H. de Boer, W.L.J. Hinrichs, Devices and formulations for pulmonary vaccination. Expert Opin. Drug Deliv. 10, 1383-1397 (2013). https://doi. org/10.1517/17425247.2013.810622

11. N. Wang, T. Wang, M. Zhang, R. Chen, R. Niu, Y. Deng, Mannose derivative and lipid A dually decorated cationic liposomes as an effective cold chain free oral mucosal vaccine adjuvant-delivery system. Eur. J. Pharm. Biopharm. 88, 194-206 (2014). https://doi. org/10.1016/j.ejpb.2014.04.007

12. N. Wang, Y. Zhen, Y. Jin, X. Wang, N. Li, S. Jiang, T. Wang, Combining different types of multifunctional liposomes loaded with ammonium bicarbonate to fabricate microneedle arrays as a vaginal mucosal vaccine adjuvant-dual delivery system (VADDS). J. Control. Release. 246, 12-29 (2017). https://doi.org/10.1016/j. jconrel.2016.12.009

13. N. Wang, T. Wang, M. Zhang, R. Chen, Y. Deng, Using procedure of emulsification-lyophilization to form lipid A-incorporating cochleates as an effective oral mucosal vaccine adjuvant-delivery system (VADS). Int. J. Pharm. 468, 39-49 (2014). https://doi. org/10.1016/j.ijpharm.2014.04.002

14. N. Wang, M. Chen, T. Wang, Liposomes used as a vaccine adjuvant-delivery system: from basics to clinical immunization. J. Control. Release. 303, 130-150 (2019). https://doi.org/10.1016/j. jconrel.2019.04.025

15. B. Corthésy, G. Bioley, Lipid-based particles: versatile delivery systems for mucosal vaccination against infection. Front. Immunol. 9(431), 1-20 (2018). https://doi.org/10.3389/fimmu .2018 .00431

16. D.E. Bloom, D. Cadarette, Infectious disease threats in the twentyfirst century: strengthening the global response. Front. Immunol. 10, 549 (2019). https://doi.org/10.3389/fimmu.2019.00549

17. E. De Wit, N. Van Doremalen, D. Falzarano, V.J. Munster, SARS and MERS: recent insights into emerging coronaviruses. Nat. Rev. Microbiol. 14, 523-534 (2016). https://doi.org/10.1038/nrmic ro.2016.81

18. M.P. Girard, J.S. Tam, O.M. Assossou, M.P. Kieny, The 2009 A (H1N1) influenza virus pandemic: a review. Vaccine. 28, 48954902 (2010). https://doi.org/10.1016/j.vaccine.2010.05.031

19. N. Zhu, D. Zhang, W. Wang, X. Li, B. Yang, J. Song, X. Zhao, B. Huang, W. Shi, R. Lu, P. Niu, F. Zhan, X. Ma, D. Wang, W. $\mathrm{Xu}, \mathrm{G}$. Wu, G.F. Gao, W. Tan, A novel coronavirus from patients with pneumonia in China. N. Engl. J. Med. 382(2020), 727-733 (2019). https://doi.org/10.1056/NEJMoa2001017

20. C. Huang, Y. Wang, X. Li, L. Ren, J. Zhao, Y. Hu, L. Zhang, G. Fan, J. Xu, X. Gu, Z. Cheng, T. Yu, J. Xia, Y. Wei, W. Wu, X. Xie, W. Yin, H. Li, M. Liu, Y. Xiao, H. Gao, L. Guo, J. Xie, G. Wang, R. Jiang, Z. Gao, Q. Jin, J. Wang, B. Cao, Clinical features of patients infected with 2019 novel coronavirus in Wuhan, China. Lancet (London, England). 6736, 1-10 (2020). https://doi. org/10.1016/S0140-6736(20)30183-5

21. J. Cohen, K. Kupferschmidt, Strategies shift as coronavirus pandemic looms. Science 367, 962-964 (2020)

22. B. Shanmugaraj, A. Malla, W. Phoolcharoen, Emergence of novel coronavirus 2019-nCoV: need for rapid vaccine and biologics development. Pathogens. 9, 1-10 (2020). https://doi.org/10.3390/ pathogens 9020148

23. B.S. Graham, J.R. Mascola, A.S. Fauci, Novel vaccine technologies essential components of an adequate response to emerging viral diseases. JAMA 319, 1431-1432 (2018). https://doi. org/10.1001/jama.2018.0345

24. T. Gargett, B. Grubor-Bauk, D. Miller, T. Garrod, S. Yu, S. Wesselingh, A. Suhrbier, E.J. Gowans, Increase in DNA vaccine efficacy by virosome delivery and co-expression of a 
cytolytic protein. Clin Transl Immunol. 3, e18 (2014). https:// doi.org/10.1038/cti.2014.13

25. P.M. Moyle, I. Toth, Modern subunit vaccines: development, components, and research opportunities. ChemMedChem 8, 360-376 (2013). https://doi.org/10.1002/cmdc.201200487

26. T.R.F. Smith, K. Schultheis, K.E. Broderick, Nucleic acid-based vaccines targeting respiratory syncytial virus: delivering the goods. Hum. Vaccines Immunother. 13, 2626-2629 (2017). https ://doi.org/10.1080/21645515.2017.1363134

27. T. Ura, K. Okuda, M. Shimada, Developments in viral vector-based vaccines. Vaccines. 2, 624-641 (2014). https://doi. org/10.3390/vaccines2030624

28. N. Wang, R. Qian, T. Liu, T. Wu, T. Wang, Nanoparticulate carriers used as vaccine adjuvant delivery systems. Crit. Rev. Ther. Drug Carrier Syst. 36, 449-484 (2019). https://doi.org/10.1615/ CritRevTherDrugCarrierSyst.2019027047

29. S. Al-Halifa, L. Gauthier, D. Arpin, S. Bourgault, D. Archambault, Nanoparticle-based vaccines against respiratory viruses. Front. Immunol. 10, 22 (2019). https://doi.org/10.3389/fimmu .2019 .00022

30. A.T. Glenny, C.G. Pope, H. Waddington, U. Wallace, The antigenic value of toxoid precipitated by potassium alum. J Pathol Bacteriol. 29, 38-39 (1926). https://doi.org/10.1002/path.17002 90106

31. Y. Wen, Y. Shi, Alum: an old dog with new tricks. Emerg. Microbes Infect. 5, e25 (2016). https://doi.org/10.1038/ emi. 2016.40

32. S.L. Hem, H. HogenEsch, Relationship between physical and chemical properties of aluminum-containing adjuvants and immunopotentiation. Expert Rev. Vaccines. 6, 685-698 (2007). https://doi.org/10.1586/14760584.6.5.685

33. E. Shardlow, M. Mold, C. Exley, Unraveling the enigma: Elucidating the relationship between the physicochemical properties of aluminium-based adjuvants and their immunological mechanisms of action, Allergy. Asthma Clin. Immunol. 14, 80 (2018). https://doi.org/10.1186/s13223-018-0305-2

34. H. Li, Y. Li, J. Jiao, H.-M.M. Hu, Alpha-alumina nanoparticles induce efficient autophagy-dependent cross-presentation and potent antitumour response. Nat. Nanotechnol. 6, 645-650 (2011). https://doi.org/10.1038/nnano.2011.153

35. B. Sun, Z. Ji, Y.-P. Liao, M. Wang, X. Wang, J. Dong, C.H. Chang, R. Li, H. Zhang, A.E. Nel, T. Xia, Engineering an effective immune adjuvant by designed control of shape and crystallinity of aluminum oxyhydroxide nanoparticles. ACS Nano 7, 10834-10849 (2013). https://doi.org/10.1021/nn404211j

36. A.P. Khandhar, H. Liang, A.C. Simpson, S.G. Reed, D. Carter, C.B. Fox, M.T. Orr, Physicochemical structure of a polyacrylic acid stabilized nanoparticle alum (nanoalum) adjuvant governs TH1 differentiation of CD4+ T cells. Nanoscale. 12, 2515-2523 (2020). https://doi.org/10.1039/c9nr09936k

37. M.T. Orr, A.P. Khandhar, E. Seydoux, H. Liang, E. Gage, T. Mikasa, E.L. Beebe, N.D. Rintala, K.H. Persson, A. Ahniyaz, D. Carter, S.G. Reed, C.B. Fox, Reprogramming the adjuvant properties of aluminum oxyhydroxide with nanoparticle technology. NPJ Vaccines. 4, 1 (2019). https://doi.org/10.1038/ s41541-018-0094-0

38. N. Wang, C. Qiu, M. Chen, T. Liu, T. Wang, Covering aluminum oxide nanoparticles with biocompatible materials to efficiently deliver subunit vaccines. Vaccines (Basel). 7, 52 (2019). https:// doi.org/10.3390/vaccines7020052

39. T. Wang, Y. Zhen, X. Ma, B. Wei, N. Wang, Phospholipid bilayercoated aluminum nanoparticles as an effective vaccine adjuvantdelivery system. ACS Appl. Mater. Interfaces. 7, 6391-6396 (2015). https://doi.org/10.1021/acsami.5b00348

40. M.B. Lutz, N. Kukutsch, A.L.J. Ogilvie, S. Rossner, F. Koch, N. Romani, G. Schuler, An advanced culture method for generating large quantities of highly pure dendritic cells from mouse bone marrow. J. Immunol. Methods. 223, 77-92 (1999). https://doi. org/10.1016/s0022-1759(98)00204-x

41. E.N. Spoelstra, C. Ince, A. Koeman, V.M. Emons, L.A. Brouwer, M.J.A. Van Luyn, B.H.C. Westerink, R. Remie, A novel and simple method for endotracheal intubation of mice. Lab. Anim. 41, 128-135 (2007). https://doi.org/10.1258/002367707779399400

42. I.S. Grewal, R.A. Flavell, The role of CD40 ligand in costimulation and T-cell activation. Immunol. Rev. 153, 85-106 (1996). https://doi.org/10.1111/j.1600-065x.1996.tb00921.x

43. N. Porciello, L. Tuosto, CD28 costimulatory signals in T lymphocyte activation: emerging functions beyond a qualitative and quantitative support to TCR signalling. Cytokine Growth Factor Rev. 28, 11-19 (2016). https://doi.org/10.1016/j.cytogfr.2016.02.004

44. K.H. Kim, Y.T. Lee, H.S. Hwang, Y.M. Kwon, Y.J. Jung, Y. Lee, J.S. Lee, Y.N. Lee, S. Park, S.M. Kang, Alum adjuvant enhances protection against respiratory syncytial virus but exacerbates pulmonary inflammation by modulating multiple innate and adaptive immune cells. PLoS ONE 10, e0139916 (2015). https://doi. org/10.1371/journal.pone.0139916

45. I. Nyoman Mantik Astawa, I.B.M. Oka, I. Made Dwinata, Antibody immunoglobulin and immunoglobulin responses against some cystic fluid proteins Cysticercus bovis in Balb/c mice. Vet. World 11, 1641-1647 (2018). https://doi.org/10.14202/vetwo rld.2018.1641-1647

46. T.L. Flach, G. Ng, A. Hari, M.D. Desrosiers, P. Zhang, S.M. Ward, M.E. Seamone, A. Vilaysane, A.D. Mucsi, Y. Fong, E. Prenner, C.C. Ling, J. Tschopp, D.A. Muruve, M.W. Amrein, Y. Shi, Alum interaction with dendritic cell membrane lipids is essential for its adjuvanticity. Nat. Med. 17, 479 (2011)

47. J. Wang, P. Li, Y. Yu, Y. Fu, H. Jiang, M. Lu, Z. Sun, S. Jiang, L. Lu, M.X. Wu, Pulmonary surfactant-biomimetic nanoparticles potentiate heterosubtypic influenza immunity. Science 367, 810 (2020). https://doi.org/10.1126/science.aau0810

48. T.P. Velavan, C.G. Meyer, The COVID-19 epidemic. Trop. Med. Int. Heal. 25, 278-280 (2020). https://doi.org/10.1111/tmi.13383

49. M.F. Bachmann, G.T. Jennings, Vaccine delivery: a matter of size, geometry, kinetics and molecular patterns. Nat. Rev. Immunol. 10, 787-796 (2010). https://doi.org/10.1038/nri2868

50. C. Exley, P. Siesjo, H. Eriksson, The immunobiology of aluminium adjuvants: how do they really work? Trends Immunol. 31, 103-109 (2010). https://doi.org/10.1016/j.it.2009.12.009

51. S. Rauch, E. Jasny, K.E. Schmidt, B. Petsch, New vaccine technologies to combat outbreak situations. Front. Immunol. 9, 1963 (2018). https://doi.org/10.3389/fimmu.2018.01963

52. R. Li, S. Pei, B. Chen, Y. Song, T. Zhang, W. Yang, J. Shaman, Substantial undocumented infection facilitates the rapid dissemination of novel coronavirus (SARS-CoV2). Science 3221, 1-9 (2020). https://doi.org/10.1126/science.abb3221

53. J.A. Whitsett, S.E. Wert, T.E. Weaver, Alveolar surfactant homeostasis and the pathogenesis of pulmonary disease. Annu. Rev. Med. 61, 105-119 (2010). https://doi.org/10.1146/annur ev.med.60.041807.123500

54. S.H. Han, R.K. Mallampalli, The role of surfactant in lung disease and host defense against pulmonary infections. Ann. Am. Thorac. Soc. 12, 765-774 (2015). https://doi.org/10.1513/Annal sATS.201411-507FR

55. D. Mizuno, M. Ide-Kurihara, T. Ichinomiya, I. Kubo, H. Kido, Modified pulmonary surfactant is a potent adjuvant that stimulates the mucosal iga production in response to the influenza virus antigen. J. Immunol. 176, 1122-1130 (2006). https://doi.org/10.4049/ jimmunol.176.2.1122

Publisher's Note Springer Nature remains neutral with regard to jurisdictional claims in published maps and institutional affiliations. 


\section{Affiliations}

\section{Ning Wang ${ }^{1} \cdot$ Chunliu Wei ${ }^{2} \cdot$ Zina Zhang ${ }^{2} \cdot$ Ting Liu $^{2} \cdot$ Ting Wang $^{2}$ (I)}

1 School of Food and Bioengineering, Hefei University of Technology, 193 Tun Brook Road, Hefei 230009, Anhui Province, China
2 School of Pharmacy, Anhui Medical University, 81 Plum Hill Road, Hefei 230032, Anhui Province, China 